\title{
Effects of Home-based Exercise Training with Wireless Monitoring on the Left Ventricular Function of Acute Coronary Syndrome Patients
}

\author{
Younghwa Lee, PhD, $\mathrm{PT}^{1)}$, Jaehong Lee, PhD, PT ${ }^{1)^{*}}$, Hyunkyu Seo, PhD, $\mathrm{PT}^{1)}$, \\ Kyoung Kim, PhD, $\mathrm{PT}^{2)}$, Dongki Min, MS, PT ${ }^{3)}$, Jinhwan Lee, MS, PT ${ }^{4}$, Jeonghee Choi, MS $^{5)}$ \\ 1) Department of Physical Therapy, Daegu Health College: 15 Youngsongro, Buk-gu, Daegu 702-722, \\ Republic of Korea. TEL: +82 53-320-4512, FAX: +82 53-320-1350 \\ 2) Department of Physical Therapy, College of Rehabilitation Science, Daegu University \\ 3) Department of Rehabilitation Medicine, Graduate school of medicine, Keimyung University \\ 4) Major in Physical Therapy, Department of Rehabilitation Science, Graduate School, Daegu \\ University \\ 5) Department of Nursing, Kyongbuk Science College
}

\begin{abstract}
Purpose] While recent studies have reported that cardiac rehabilitation is an effective treatment, there have been few studies of its effects in rehabilitation for acute coronary syndrome (ACS). Therefore, the purpose of this study was to identify the effects of a home-based exercise training using a wireless electrocardiogram (ECG) monitoring device on ACS patients. [Subjects] Fifty ACS patients were randomly divided into a experimental group of 25 patients and a control group of 25 patients. [Methods] The experimental group received education on the training before discharge from hospital and started home-based exercise training two weeks after discharge from hospital. The control group received conventional treatments. The left ventricular function was measured in both groups before the intervention at and 12 weeks, at the end of the intervention. [Results] Both the experimental group and the control group showed significant improvements in the left ventricular ejection fraction and number of regional wall motion abnormalities. In the comparison of the two groups, the experimental group showed a significantly greater decline in regional wall motion abnormalities than the control group. [Conclusion] Home-based exercise training implemented with a wireless monitoring device was effective at improving the left ventricular function of ACS patients.

Key words: Acute coronary syndrome (ACS), Home-based exercise, Ventricular function
\end{abstract}

(This article was submitted Dec. 11, 2012, and was accepted Jan. 17, 2013)

\section{INTRODUCTION}

The numbers of patients with circulatory disorders are gradually increasing in Korea and the rest of the world due to health-related factors such as increased intake of animal fat, increased stress levels, decreased amount of exercise, increases in obesity, and an aging population. Every year, Korea also experiences increases in the numbers and the death rates of patients with ischemic heart disease (IHD $)^{1)}$. Cardiac rehabilitation, which has an important part in the overall care of patients with acute coronary syndrome (ACS), has recently been actively implemented as an intervention for IHD ${ }^{2,3)}$. A study of IHD patients after cardiac rehabilitation within a hospital demonstrated statistically significant changes in their left ventricular function ${ }^{4}$.

Despite these effects of cardiac rehabilitation, patients who participate in such treatment frequently give up during the course of the treatment for various reasons, indication there are a number of issues with compliance in cardiac re-

*To whom correspondence should be addressed. E-mail: heart0630@dhc. ac.kr habilitation ${ }^{5)}$. Thus, patients requiring cardiac rehabilitation require methods suited to home-based rehabilitation, with the exercise intensity and protocols prescribed by hospitals tailored to the needs of individual patients. Studies of the ability of cardiac rehabilitation to treat ACS, which is closely related to sudden deaths, are considered more meaningful in clinical terms than previous studies on various types of IHD patients.

Therefore, the present study attempted to identify the effects of home-based exercise training with a wireless electrocardiogram (ECG) monitoring device on the left ventricular function of ACS patients.

\section{SUBJECTS AND METHODS}

The study subjects were 50 ACS patients, who were hospitalized at the Keimyung University Dongsan Center in Korea from October 2010 to March 2011, who received a percutaneous coronary intervention (PCI). The study was carried out until June 2011. Twenty-five of the subjects were randomly placed in a experimental group which received cardiac rehabilitation, and 25 subjects were placed in a con- 
Table 1. Clinical characteristics of the subjects

\begin{tabular}{lcc}
\hline Variables & $\begin{array}{r}\text { Experimental } \\
\text { group }(\mathrm{n}=25)\end{array}$ & $\begin{array}{c}\text { Control group } \\
(\mathrm{n}=25)\end{array}$ \\
\hline Male (\%) & $18(72 \%)$ & $19(76 \%)$ \\
Age (yr) & $55.56 \pm 9.23$ & $57.88 \pm 7.90$ \\
Height (cm) & $166.92 \pm 6.66$ & $166.68 \pm 8.37$ \\
Weight (kg) & $66.08 \pm 10.79$ & $69.60 \pm 13.34$ \\
Previous history & & \\
Smoking (\%) & $12(48 \%)$ & $14(56 \%)$ \\
Hypertension (\%) & $7(28 \%)$ & $10(40 \%)$ \\
Diabetes (\%) & $4(16 \%)$ & $7(28 \%)$ \\
Hyperlipidemia (\%) & $4(16 \%)$ & $4(16 \%)$ \\
Medications & & \\
ARB (\%) & $6(24 \%)$ & $6(24 \%)$ \\
ACEi (\%) & $15(60 \%)$ & $14(56 \%)$ \\
CCB (\%) & $2(8 \%)$ & $3(12 \%)$ \\
Statin (\%) & $14(56 \%)$ & $14(56 \%)$ \\
Beta-blockers (\%) & $21(84 \%)$ & $23(92 \%)$ \\
\hline
\end{tabular}

Mean \pm SD, ARB: angiotensin receptor blocker; ACEi: angiotensin converting enzyme inhibitors; $\mathrm{CCB}$ : calcium channel blocker

trol group with conventional care (Table 1). The cardiac rehabilitation program was comprised of standard mediations of conventional treatment, education, and exercise training.

The selection criteria were individuals aged 20 to 70 years with a diagnosis of ACS who had received a PCI. Candidates with chronic stable angina, New York Heart Association class III-IV, a left ventricular ejection fraction of less than $30 \%$, chronic renal failure, or exercise disabilities were excluded from the study.

The details of the study were explained to the subjects during their initial hospitalization, and each patient read and signed the informed consent from approved by the institutional medical review board.

A researcher who read and understood the educational content of the intervention, including the risk factors for cardiac diseases, and the application of diet therapy and exercise training, educated the subjects before they were discharged from hospital. The subjects participated in the exercise program from the second week after their hospital discharge to the 12th week. The exercise program consisted of a walking exercise four times a week. Each exercise included a warm-up and a cool-down of flexibility exercises, each lasting $10 \mathrm{~min}$, and the main walking exercise which lasted for for 30 minutes.

When the subjects performed the exercise at home, they were instructed to wear a wireless ECG monitoring device (HeartCall $^{\mathrm{TM}}$, U-HEART, Korea) provided two weeks after their hospital discharge. Thereafter, their ECG and heart rates were measured. The researcher also conducted individual consultations with the patients over the phone at least once a week to control risk factors, and to adjust the exercise intensity depending on the phase of the rehabilitation program.

The exercise intensity was calculated in terms of heart rate reserve (HRR) based on the maximum heart rate (HR $\max$ ) and the resting heart rate (HR rest) measured through a gradual symptom-limited exercise load test. The HHR was increased from $40 \%$ to $80 \%$ in phases. The target HR was calculated using the Karvonen formula: target heart rate $=(\mathrm{HR} \max -\mathrm{HR}$ rest $) \times($ percentage $\%)+$ HR rest. The control group was treated with standard medications and dietary therapy for conventional treatments and performed home-based individual exercises. This group did not participate in the cardiac rehabilitation program.

Both the experimental group and the control group underwent gradual symptom-limited exercise load tests before the intervention and at 12 weeks, at the end of the intervention. The subjects' exercise ability was measured in a gradual symptom-limited exercise load test, based on a modified Bruce protocol, performed on a treadmill (Marquette, GE, USA). In the second minute of each exercise phase, the HR and blood pressure were measured using a 12-channel realtime ECG machine and an automatic blood pressure monitor (Ambulatory Blood Pressure, GE, USA).

Before the intervention and at 12 weeks, at the end of the intervention, both the experimental and the control groups underwent transthoracic echocardiography (Vivid7, GE, USA). From this, the left ventricular end-diastolic dimension, left ventricular end-systolic dimension, left ventricular ejection fraction, and regional wall motion abnormalities were measured.

Statistical analyses were performed using SPSS for Windows version 12.0 (SPSS, Chicago, IL, USA). The paired t-test was conducted to examine the significance of within group changes in the left ventricular function resulting from the intervention. To verify differences between the two groups, ANCOVA tests were conducted. The statistical significance level was chosen as $\mathrm{p}<0.05$.

\section{RESULTS}

The left ventricular ejection fraction of the experimental group exhibited a statistically significant increase from $52.48 \pm 9.40 \%$ before the intervention to $62.04 \pm 9.42 \%$ at 12 weeks, at the end of the intervention $(\mathrm{p}<0.05)$. In this group, the number of regional wall motion abnormalities showed a statistically significant decrease from $1.32 \pm 0.39$ before the intervention to $1.08 \pm 0.17$ at 12 weeks, at the end of the intervention $(p<0.05)$. The left ventricular ejection fraction of the control group exhibited a statistically significant increase from $52.12 \pm 8.32 \%$ before the intervention to $58.64 \pm 9.34 \%$ at 12 weeks, at the end of the intervention ( $p$ $<0.05$ ). The number of regional wall motion abnormalities of the control group also showed a statistically significant decline from $1.37 \pm 0.36$ before the intervention to $1.29 \pm$ 0.32 at 12 weeks, at the end of the intervention $(p<0.05)$. In the comparison of regional wall motion abnormalities between the two groups, the experimental group showed a statistically more significant decline than the control group $(\mathrm{p}<0.05)$ (Table 2).

\section{DISCUSSION}

Cardiac rehabilitation has been reported to help patients 
Table 2. Echocardiography data of the experimental and control groups

\begin{tabular}{lcccc}
\hline \multirow{2}{*}{ Variables } & \multicolumn{2}{c}{ Experimental group $(\mathrm{n}=25)$} & \multicolumn{2}{c}{ Control group $(\mathrm{n}=25)$} \\
& Baseline & After 12 wk & \multicolumn{1}{c}{ Baseline } & After 12 wk \\
\hline LVDd $(\mathrm{cm})$ & $5.05 \pm 0.38$ & $4.90 \pm 0.81$ & $5.06 \pm 0.62$ & $5.15 \pm 0.45$ \\
LVDs $(\mathrm{cm})$ & $3.51 \pm 0.43$ & $3.56 \pm 0.69$ & $3.50 \pm 0.68$ & $3.45 \pm 0.73$ \\
LVEF $(\%)$ & $52.48 \pm 9.40$ & $62.04 \pm 9.42^{*}$ & $52.12 \pm 8.32$ & $58.64 \pm 9.34^{*}$ \\
RWMA score & $1.32 \pm 0.39$ & $1.08 \pm 0.17^{*}$ & $1.37 \pm 0.36$ & $1.29 \pm 0.32^{*}$ \\
\hline
\end{tabular}

Mean $\pm \mathrm{SD}, * \mathrm{p}<0.05$ : comparison between baseline and $12 \mathrm{wk}$ follow-up, $* * \mathrm{p}<0.05$ : significant difference, experimental group versus control group, LVDd: left ventricular end-diastolic dimension; LVDs: left ventricular end-systolic dimension; LVEF: left ventricular ejection fraction; RWMA: regional wall motion abnormality

with cardiac diseases recover quickly and reduce risk factors associated with reoccurrence. Interventions include exercise to reduce coronary risk factors and medications to prevent coronary artery diseases and sudden cardiac deaths. In addition, the purpose of cardiac rehabilitation is to induce various physiological adaptations in the heart, skeletal muscles, and blood vessels, increasing oxygen content within the arteries during maximal exercises. Some studies have recommended that regular exercise training should, in most cases, be prescribed based on individual programs for primary and secondary interventions ${ }^{6-8)}$.

In the present study, the echocardiography results confirmed a statistically significant increase in the left ventricular ejection fraction of both groups. However, the experimental group attained a more significant increase, on average, than the control group. Also, the number of regional wall motion abnormalities in the experimental group exhibited a statistically more significant decline than the control group.

In both groups, the improvements in the left ventricular ejection fraction can be inferred as having been influenced by the PCI and the medications, as well as the exercise training. However, given that the scores of regional wall motion abnormalities showed a more statistically significant decrease in the experimental group, the exercise training can be considered to have been helpful in reducing cardiovascular blood circulation disorders resulting from regional wall motion abnormalities.

A previous study that conducted exercise training for patients with coronary artery diseases reported statistically significant improvements in blood circulation ${ }^{9}$. In a study of IHD patients treated with cardiac rehabilitation, Ahn et al. ${ }^{4)}$ reported that their patients showed a statistically significant increase in the left ventricular ejection fraction. However, they detected no statistically significant declines in the left ventricular end-diastolic diameter, the left ventricular end-systolic diameter, and the score of regional wall motion abnormalities. Andres et al. ${ }^{10)}$ reported that cardiac rehabilitation can increase heart disease patients' blood volumes at the left-ventricular end-diastolic and improve cardiac outputs, as well as increasing maximum oxygen consumption, thus slowing the progress of heart disease.

The findings discussed above are similar to the results of the present study of a statistically significant increase in the left ventricular ejection fraction. However, the fact that the present study also observed a statistically significant decline in the scores of regional wall motion abnormalities may be meaningful. Based on the present results, we conclude that stepwise individually prescribed exercises can increase patients' maximum oxygen consumption and reduce their myocardial burdens, thereby increasing leftventricle ejection fractions and reducing the occurrence of regional wall motion abnormality. To achieve more significant improvements through exercise training, however, an extended research period with a greater number of subjects should be undertaken.

In conclusion, the present study of ACS patients with IHD found statistically significant improvements in left ventricular function following the implementation of a home-based exercise training program shortly after the subjects were discharged from the hospital. The anxiety that patients generally feel during exercising at home was reduced by wearing the wireless monitoring device in this study, and the results of this study can be considered a clinically meaningful. The findings of this study will need to be confirmed through future studies based on additional cases of clinical utilization of the protocol described in this study.

\section{REFERENCES}

1) Statistics Korea: Statistics of cause of death. Mortality of chronic disease. http://www.index.go.kr/egarns/stts/jsp/potal/stts/PO_STTS_IdxMain. jsp?idx cd $=1438$ (Accessed Sep. 10, 2010).

2) Brubaker PH, Kaminsky LA, Whaley MH: Coronary artery disease. Essentials of Prevention and Rehabilitation Programs. Champaign: Human Kinetics press, 2010, pp 171-483.

3) Vonder Muhll I, Daub B, Black B, et al.: Benefits of cardiac rehabiltiation in the ninth decade of life in patients with coronary heart disease. Am J Cardiol, 2002, 90: 645-648. [Medline] [CrossRef]

4) Ahn JK, Kim C, Bang IK, et al.: Effectiveness of cardiac rehabilitation on exercise capacity and ventricular function in ischemic heart disease patients. J Korean Sports Med, 2006, 24: 229-236.

5) Kim C, Lim HS, Ahn JK, et al.: The reasons that cardiac patients did not participate in and drop out from the cardiac rehabilitation program. J Korean Acad Rehab Med, 2002, 26: 790-796.

6) Bethell HJ: Exercise-based cardiac rehabilitation. Medicine, 2006, 34: 195-196. [CrossRef]

7) Adres P: Cardiac rehabilitation and secondary prevention of coronary heart disease. N Engl J Med, 2001, 345: 892-902. [Medline] [CrossRef]

8) Joshi SB: Exercise training in the management of cardiac failure and ischaemic heart disease. Heart Lung Circ, 2007, 16: S83-S87. [Medline] [CrossRef]

9) Hambrecht R, Wolf A, Gielen S, et al.: Effect of exercise on coronary endothelial function in patients with coronary artery disease. N Engl J Med, 2000, 342: 454-460. [Medline] [CrossRef]

10) Digenio AG, Noakes TD, Joughin $H$, et al.: Effect of myocardial ischemia on left ventricular function and adaptability to exercise training. Med Sci Sports Exerc, 1999, 31: 1094-1101. [Medline] [CrossRef] 